\title{
SERCA Inhibition Limits the Functional Effects of Cyclic GMP in Both Control and Hypertrophic Cardiac Myocytes
}

\author{
Qihang Zhang ${ }^{a}$ Tomer Davidov $^{b}$ Harvey R. Weiss ${ }^{a}$ Peter M. Scholz ${ }^{b}$ \\ Heart and Brain Circulation Laboratory, Departments of a Physiology and Biophysics and b ${ }^{\text {Surgery, }}$ \\ UMDNJ-Robert Wood Johnson Medical School, Piscataway, N.J., USA
}

\section{Key Words}

Cardiac myocytes $\cdot$ Myocyte function $\cdot$ Calcium transients •

Cyclic GMP · Thapsigargin · Cyclopiazonic acid

\begin{abstract}
The negative functional effects of cyclic GMP are controlled by the sarcoplasmic reticulum calcium-ATPase (SERCA). The effects of cyclic GMP are blunted in cardiac hypertrophy. We tested the hypothesis that the interaction between cyclic GMP and SERCA would be reduced in hypertrophic cardiac myocytes. Myocytes were isolated from 7 control and 7 renal-hypertensive hypertrophic rabbits. Control and hypertrophic myocytes received 8-bromo-cGMP (8-Br-cGMP; $10^{-7}$, $\left.10^{-6}, 10^{-5} \mathrm{~mol} / \mathrm{l}\right)$, the SERCA blocker thapsigargin $\left(10^{-8} \mathrm{~mol} / \mathrm{l}\right)$ followed by 8-Br-cGMP, or the SERCA blocker, cyclopiazonic acid (CPA; $\left.10^{-7} \mathrm{~mol} / \mathrm{l}\right)$ followed by 8 -Br-cGMP. Percent shortening and maximal rate of shortening and relaxation were recorded using a video edge detector. Changes in cytosolic $\mathrm{Ca}^{2+}$ were assessed in fura 2-loaded myocytes. In controls, 8-Br-cGMP caused a significant $36 \%$ decrease in percent shortening from $5.8 \pm 0.4$ to $3.7 \pm 0.3 \%$. Thapsigargin and CPA did not affect basal control or hypertrophic myocyte function. When 8-Br-cGMP was given following thapsigargin or CPA, the negative effects of 8-Br-cGMP on control myocyte function were reduced. In hypertrophic myocytes, 8-Br-cGMP caused a smaller but significant $17 \%$ decrease in percent shortening from $4.7 \pm 0.2$ to $3.9 \pm 0.1 \%$. When 8 -
\end{abstract}

Br-cGMP was given following thapsigargin or CPA, no significant changes occurred in hypertrophic cell function. Intracellular $\mathrm{Ca}^{2+}$ transients responded in a similar manner to changes in cell function in control and hypertrophic myocytes. These results show that the effects of cyclic GMP were reduced in hypertrophic myocytes, but this was not related to SERCA. In presence of SERCA inhibitors, the responses to cyclic GMP were blunted in hypertrophic as well as control myocytes.

Copyright $\odot 2009$ S. Karger AG, Basel

\section{Introduction}

Cyclic GMP, produced in response to nitric oxide and natriuretic peptides, is an important regulator of myocardial function. It has been shown that cyclic GMP reduces myocardial metabolism, inotropy and function $[1,2]$. Elevation in cyclic GMP decreases myocyte oxygen consumption and myocyte contraction in rabbit, mouse and dog hearts and cardiac ventricular myocytes [3-6]. The negative effects of cyclic GMP are partly mediated through the cyclic GMP-dependent protein kinase and this can reduce intracellular $\mathrm{Ca}^{2+}$ transients by activation of the sarcoplasmic reticulum calcium-ATPase (SERCA) $[7,8]$. The interaction between cyclic GMP and SERCA may be related to cyclic GMP-mediated phosphorylation of phospholamban. The cyclic GMP-signaling pathway is 
also mediated by protein kinase-independent interactions, such as cyclic GMP-gated cation channels and certain phosphodiesterases [9-11].

Cardiac hypertrophy can develop as a basic response to persistent increases in blood pressure and is induced by a variety of diseases, such as hypertension, valvular diseases, myocardial infarction, and endocrine disorders $[12,13]$. Nitric oxide and natriuretic peptides have been shown to inhibit cardiac hypertrophy via changes in cyclic GMP [11]. Increases in myocardial cyclic GMP levels have been reported in some forms of cardiac hypertrophy $[14,15]$. Previous studies from our laboratory have shown that the basal level of cyclic GMP in renal hypertensive (one-kidney, one-clip, 1K1C) rabbits was similar to control values [16]. The functional responses to cyclic GMP tend to be reduced in hypertrophic myocytes. Cyclic GMP-dependent protein kinase activity is reduced in hypertrophic cardiac myocytes $[5,17]$. Since cyclic GMPinduced reductions in cardiac myocyte function are partially mediated by activation of the sarcoplasmic reticulum $\mathrm{Ca}^{2+}$-ATPase [8], it is crucial to determine whether the importance of SERCA changes in hypertrophy.

We tested the hypothesis that the interaction between cyclic GMP and SERCA would be reduced in hypertrophic cardiac myocytes. This interaction was compared in control and 1K1C hypertrophic rabbit ventricular myocytes. We used thapsigargin and cyclopiazonic acid (CPA) to specifically inhibit SERCA activity and to reduce the negative effects of cyclic GMP on cardiac myocyte function. The cyclic GMP analog 8-bromo-cGMP (8-Br-cGMP) was used to increase intracellular cyclic GMP levels. We examined changes in ventricular myocyte function and intracellular calcium transients in these rabbit ventricular myocytes. We found reduced functional and calcium transient responses to cyclic GMP in 1K1C myocytes, but this was not related to changes in SERCA activity.

\section{Materials and Methods}

The investigation was conducted in accordance with the Guide for the Care of Laboratory Animals (DHHS Publication 85-23, revised 1996) and was approved by our Institutional Animal Care and Use Committee.

\section{K1C Hypertrophic Heart Model}

New Zealand white rabbits $(2-3 \mathrm{~kg})$ were prepared as a $1 \mathrm{~K} 1 \mathrm{C}$ renal hypertensive model under sterile, anesthetized conditions (30 mg/kg, sodium pentobarbital i.v.) as previously described [16]. A left flank incision was used to expose the left kidney, and the renal artery was carefully dissected. A sterling silver clip $(0.5-\mathrm{mm}$ gap opening) was threaded around the artery and folded over it- self, securing it in place. The incision was closed. The right kidney was then exposed through a right flank incision and the ureter, renal artery, and renal vein were ligated. The kidney was removed and the incision closed. The animals were allowed to recover for 35 days.

\section{Ventricular Myocyte Dissociation}

Ventricular myocytes were isolated from hearts of New Zealand white rabbits $(\mathrm{n}=28,2.5-3.5 \mathrm{~kg})$ as previously described [8]. Briefly, the rabbits were anesthetized with sodium pentobarbital (35 mg/kg) followed by the administration of heparin (10 units/g body weight) intravenously using the circumflex ear vein. The heart was immediately removed after an overdose of pentobarbital $(60 \mathrm{mg} / \mathrm{kg})$ and retrograde perfused through the aorta with minimal essential medium (MEM, Sigma) supplemented with 10 $\mathrm{mmol} / \mathrm{l}$ taurine, $2 \mathrm{mmol} / \mathrm{l} \mathrm{L}$-glutamic acid and $20 \mathrm{mmol} / \mathrm{l} \mathrm{HEPES}$, $\mathrm{pH}$ 7.2. After $5 \mathrm{~min}$ of perfusion with MEM, the heart was perfused with MEM containing $0.1 \%$ type II collagenase (Worthington) for $16 \mathrm{~min}$. All perfusion media were maintained at $37^{\circ} \mathrm{C}$ and equilibrated with water-saturated oxygen.

After collagenase perfusion, the heart was removed from the perfusion apparatus and the ventricle was cut into 8-10 pieces. The tissue suspension was further treated with MEM containing $0.1 \%$ collagenase and $0.5 \%$ bovine serum albumin (BSA), fraction $\mathrm{V}$ (Sigma) at $37^{\circ} \mathrm{C}$ and gently swirled at 2 cycles/s for $5 \mathrm{~min}$. A slurry containing isolated myocytes was decanted from tissue suspension. The isolated cells were washed 3 times in MEM containing $0.5 \%$ BSA and centrifuged at low speed $(34 \mathrm{~g})$ to completely remove the collagenase and subcellular debris. Incubation of the remaining tissue with collagenase was repeated at least 2 more times. Myocyte viability was assessed by maintenance of a rodshaped morphology and was between 50 and $70 \%$. Yields were typically $10-14 \times 10^{6}$ rod-shaped cells/heart.

\section{Myocyte Functional Measurements}

Individual ventricular myocytes were studied for function. Cells were suspended in $2 \mathrm{ml}$ of $2 \mathrm{mmol} / 1 \mathrm{Ca}^{2+} \mathrm{MEM}$ solution containing $0.5 \% \mathrm{BSA}$ maintained at $37^{\circ} \mathrm{C}$ in a chamber that was fitted onto the stage of an inverted light microscope (Zeiss Axiovert 125, Carl Zeiss). Two platinum wires were inserted into two parallel sides of the chamber and were used to pace the myocytes by electric field stimulation $(1 \mathrm{~Hz}, 5 \mathrm{~ms}$ duration, voltage $10 \%$ above threshold, and polarity altered with each pulse). Unloaded shortening of selected cardiac myocytes was measured on-line by using a video edge-detector system (Crystal Biotech, model VED114, Patton Biomedical) and a camera, which detected the change of position of both edges of the cell. Data were collected continuously. The output of the video edge detector was fed into a television monitor and computer, which then analyzed the data. Cells used to determine the functional parameters were healthy and could react to different reagents throughout the course of the experiment. Cell contraction measurements were obtained on random cells in each preparation and each cell was required to complete its protocol. Untreated cells continued to contract at a constant level over the time course of the experiment.

\section{Experimental Protocol}

Ventricular myocytes were used in the following protocols for cell functional measurements. In all groups, myocytes were suspended in a chamber with $2 \mathrm{ml}$ of MEM containing $2 \mathrm{mmol} / \mathrm{Ca}^{2+}$ 
and $0.5 \%$ BSA. After a 10 -min stabilization period paced with electrical field stimulation, baseline contraction data for an individual myocyte were recorded. At 5-min intervals, reagents were added to the medium and allowed to diffuse to the cell during which cell contractility was measured. In the first group, 8 -Br-cGMP was added to myocytes at concentrations of $10^{-7}, 10^{-6}$ and $10^{-5} \mathrm{~mol} / \mathrm{l}$. In the second group, thapsigargin $\left(10^{-8} \mathrm{~mol} / \mathrm{l}\right)$ was given to myocytes before 8 -Br-cGMP treatment. In the third group, CPA $\left(10^{-7} \mathrm{~mol} / \mathrm{l}\right)$ was given to myocytes before 8 - $\mathrm{Br}$-cGMP treatment. A minimum of 10 consecutive contractions was averaged for each data point. For each protocol at least 3 cells in each animal were repeatedly measured. Measurements obtained included resting cell length, absolute cell shortening, maximal rate of shortening, maximal rate of cell relaxation, and calculated percentage of cell shortening.

\section{Intracellular $\left[\mathrm{Ca}^{2+}\right]$ Measurements}

For the measurement of intracellular $\mathrm{Ca}^{2+}$ concentration, the fluorescent $\mathrm{Ca}^{2+}$ indicator, fura-2, was used. Ventricular myocytes were incubated with $2 \mu \mathrm{mol} / \mathrm{l}$ fura-2 acetoxymethyl ester (fura-2 AM, Molecular Probes, Eugene, Oreg., USA) in MEM solution containing $0.5 \% \mathrm{BSA}$ and $1 \mathrm{mmol} / \mathrm{CaCl}_{2}$ at room temperature for $1 \mathrm{~h}$. Unincorporated fura-2 AM was removed by washing myocytes twice in fura-2 AM-free MEM solution. The fura-2-loaded cells were suspended in $2 \mathrm{ml}$ of $2 \mathrm{mmol} / \mathrm{C} \mathrm{Ca}^{2+} \mathrm{MEM}$ solution containing $0.5 \% \mathrm{BSA}$ maintained at $37^{\circ} \mathrm{C}$ in a chamber that was fitted onto the stage of a Nikon inverted microscope (TS100). Ventricular myocytes were paced by electric field stimulation at $1 \mathrm{~Hz}, 5 \mathrm{~ms}$ duration. A dual excitation spectrofluorometer was used to record fluorescence emissions (480-520 nm) elicited from exciting wavelengths at 360 and $380 \mathrm{~nm}$ and measurements recorded with an IonOptix Soft Edge and Fluorescence System (IonOptix, Milton, Mass., USA). Changes in $\left[\mathrm{Ca}^{2+}\right]_{\mathrm{i}}$ were determined from the ratio of the fluorescence intensity at $360 / 380 \mathrm{~nm}$ wavelengths. Intracellular calcium levels were measured at baseline and in the presence of cyclic GMP $10^{-5} \mathrm{~mol} / \mathrm{l}$, or at baseline and after addition of CPA $10^{-7} \mathrm{~mol} / \mathrm{l}$ followed by cyclic GMP $10^{-5} \mathrm{~mol} / \mathrm{l}$. Each experimental protocol was performed in at least three myocytes per group from each animal and 7 animals were used for each group.

\section{Statistics}

Results are expressed as mean \pm SEM. A repeated measures analysis of variance was used to compare variables measured during the experimental and control conditions. Duncan's multiple range test was used to compare the differences post hoc. This analysis was used to determine differences between the control and hypertrophic groups and the various treatments for cardiac myocyte function. Student's t tests were used to compare cell lengths and differences between baseline and cyclic GMP and CPA treatments and for the calcium measurements in control and hypertrophic myocytes. In all cases, a value of $\mathrm{p}<0.05$ was accepted as significant.

\section{Results}

To determine the degree of renal hypertension-induced cardiac hypertrophy, we measured the heart weight/body weight ratio. In the control rabbits, the heart to body weight ratio was $2.2 \pm 0.3 \mathrm{~g} / \mathrm{kg}(\mathrm{n}=7)$. In $1 \mathrm{~K} 1 \mathrm{C}$ cardiac hypertrophic rabbits, the heart weight/ body weight ratio was $2.6 \pm 0.2 \mathrm{~g} / \mathrm{kg}(\mathrm{n}=7)$, which was statistically higher than that of control rabbits. The cell length of hypertrophic myocytes $(181 \pm 5 \mu \mathrm{m}, \mathrm{n}=63)$ was also significantly longer than that of control myocytes $(157 \pm 3 \mu \mathrm{m}, \mathrm{n}=63)$. Baseline percent shortening, maximum rate of shortening and maximum rate of relaxation were all similar in control and $1 \mathrm{~K} 1 \mathrm{C}$ ventricular myocytes.

\section{Myocyte Function Was Reduced to a Greater Extent by 8-Br-cGMP in Control Compared to Hypertrophic Myocytes}

In control rabbit hearts, 8 -Br-cGMP caused a significant concentration-dependent decrease in myocyte contractility. Percent shortening was reduced by $36 \%$ from $5.8 \pm 0.4 \%$ at baseline to $3.7 \pm 0.3 \%$ in presence of $10^{-5}$ mol/l 8-Br-cGMP (fig. 1). Maximum rate of shortening was significantly reduced by $24 \%$ from $70.5 \pm 3.5$ to 53.5 $\pm 5.4 \mu \mathrm{m} / \mathrm{s}$ with 8 -Br-cGMP (fig. 2). Maximum rate of relaxation was reduced by $17 \%$ from $68.4 \pm 3.6$ to $51.9 \pm$ $5.3 \mu \mathrm{m} / \mathrm{s}$ with $10^{-5} \mathrm{~mol} / \mathrm{l} 8$-Br-cGMP (fig. 3).

In hypertrophic (1K1C) myocytes, 8-Br-cGMP caused a smaller but significant $17 \%$ decrease in percent shortening, compared to $36 \%$ in control, from $4.7 \pm 0.2$ to $3.9 \pm$ $0.1 \%$ (fig. 2). The addition of $10^{-5} \mathrm{~mol} / \mathrm{l} 8-\mathrm{Br}-\mathrm{cGMP}$ also caused a statistically significant decrease in maximum rate of shortening $(71.8 \pm 6.8$ to $63.7 \pm 5.8 \mu \mathrm{m} / \mathrm{s})$. However, the decrease in maximum rate of relaxation (68.5 \pm 6.5 to $61.9 \pm 5.7 \mu \mathrm{m} / \mathrm{s}$ ) was not significant in the hypertrophic myocytes.

\section{Inhibition of SERCA Did Not Affect Baseline Myocyte Function}

Two specific SERCA inhibitors, thapsigargin and CPA, were used to block intracellular calcium reuptake to the sarcoplasmic reticulum and to investigate the importance of SERCA in control of baseline function in control and hypertrophic myocytes. In control myocytes, thapsigargin $10^{-8} \mathrm{~mol} / \mathrm{l}$ did not significantly affect myocyte function with regard to percent shortening, maximum rate of shortening at baseline or maximum rate of relaxation at baseline in control myocytes. Similarly, in 1K1C hypertrophic myocytes, thapsigargin alone did not affect baseline myocyte function. The other SERCA inhibitor, CPA also did not affect baseline myocyte function in control or $1 \mathrm{~K} 1 \mathrm{C}$ hypertrophic ventricular myocytes (fig. 1-3). 

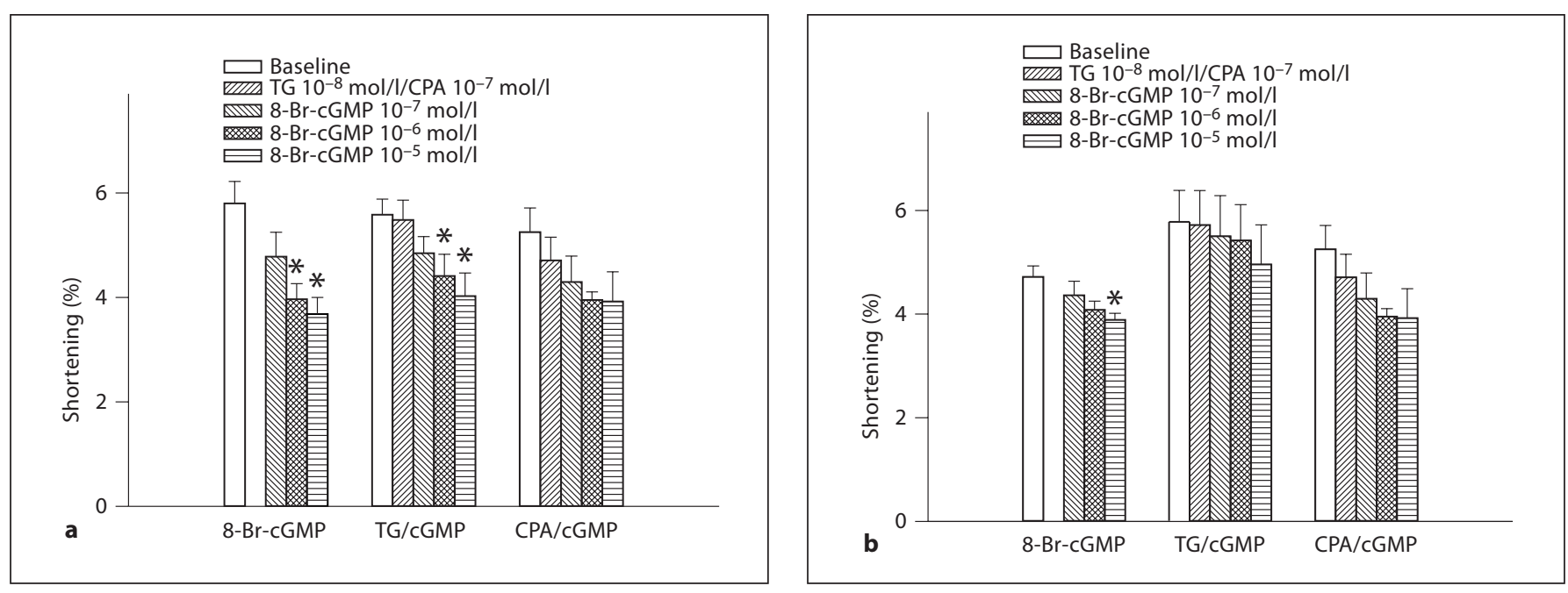

Fig. 1. The effects of 8-Br-cGMP, thapsigargin (TG) and CPA on percentage shortening of ventricular myocytes from control (a) and $1 \mathrm{~K} 1 \mathrm{C}$ hypertrophic rabbit hearts (b). In the first group, the myocytes were treated with 8 $\operatorname{Br}-\mathrm{cGMP}\left(10^{-7}, 10^{-6}, 10^{-5} \mathrm{~mol} / \mathrm{l}\right)$. The second group was treated with TG $\left(10^{-8} \mathrm{~mol} / \mathrm{l}\right)$ followed by $8-\mathrm{Br}-\mathrm{cGMP}$ $\left(10^{-7}, 10^{-6}, 10^{-5} \mathrm{~mol} / \mathrm{l}\right)$. The third group was treated with CPA $\left(10^{-7} \mathrm{~mol} / \mathrm{l}\right)$ followed by 8 -Br-cGMP $\left(10^{-7}, 10^{-6}\right.$, $\left.10^{-5} \mathrm{~mol} / \mathrm{l}\right)$. The effects of $8-\mathrm{Br}-\mathrm{cGMP}$ were less in the $1 \mathrm{~K} 1 \mathrm{C}$ myocyte. Note that SERCA inhibition reduced the effects of 8-Br-cGMP. * Significantly different from baseline.
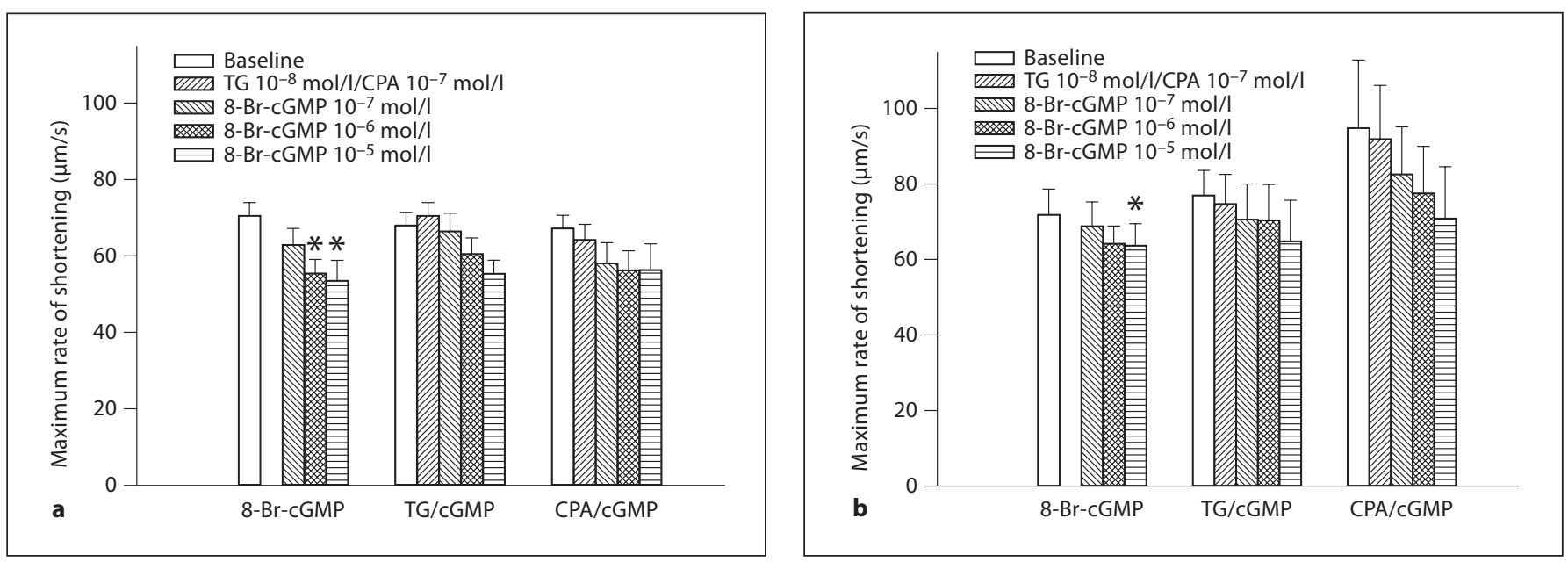

Fig. 2. The effects of 8-Br-cGMP, TG and CPA on maximum rate of shortening of ventricular myocytes from control (a) and 1K1C hypertrophic rabbit hearts (b). The effects of 8-Br-cGMP were less in the $1 \mathrm{~K} 1 \mathrm{C}$ myocyte. Note that SERCA inhibition reduced the effects of 8-Br-cGMP. * Significantly different from baseline.

SERCA Inhibition Reduced the Effects of 8-Br-cGMP on Cell Function in Both Control and Hypertrophic Myocytes

In control myocytes, when 8-Br-cGMP was administered following CPA the negative functional effects of 8$\mathrm{Br}-\mathrm{cGMP} 10^{-5} \mathrm{~mol} / \mathrm{l}$ were reduced. There were no longer significant 8-Br-cGMP-induced reductions in percent shortening, maximum rate of shortening or maximum rate of relaxation (fig. 1-3). Similarly, thapsigargin also reduced the negative functional effects of 8 -Br-cGMP on myocyte contractility. Thapsigargin prevented the $8-\mathrm{Br}-$ cGMP-induced reductions in maximum rate of shorten- 

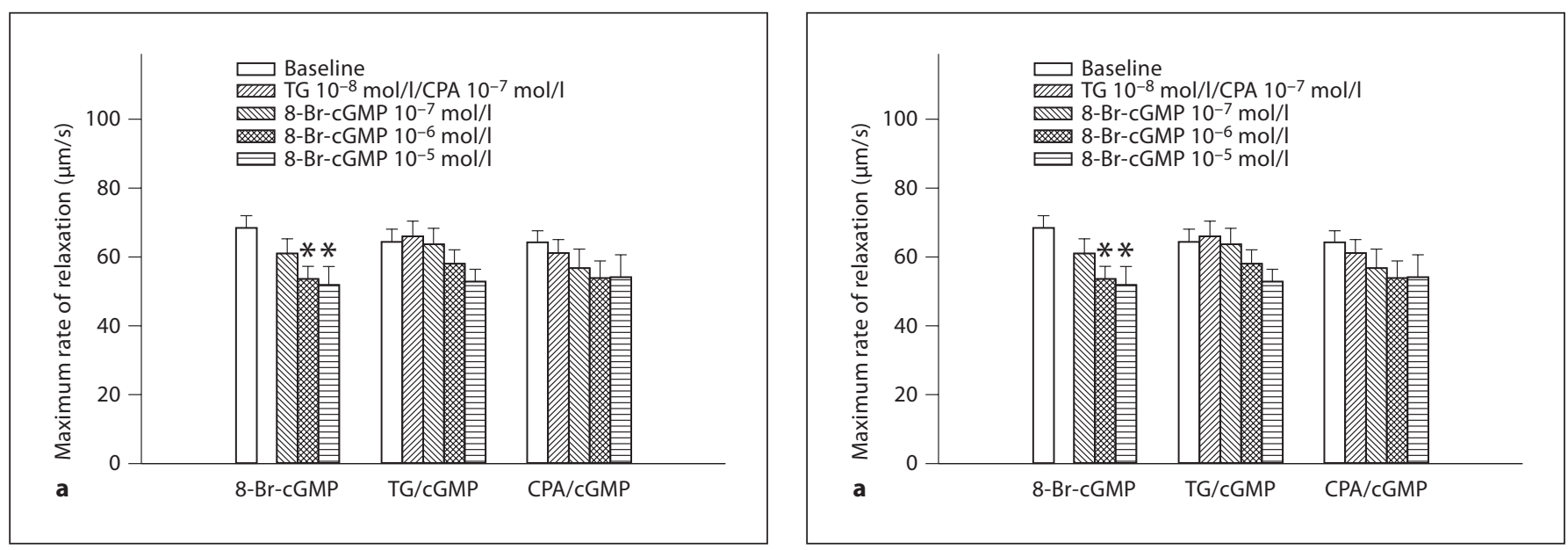

Fig. 3. The effects of 8-Br-cGMP, TG and CPA on maximum rate of relaxation of ventricular myocytes from control (a) and 1K1C hypertrophic rabbit hearts (b). * Significantly different from baseline.
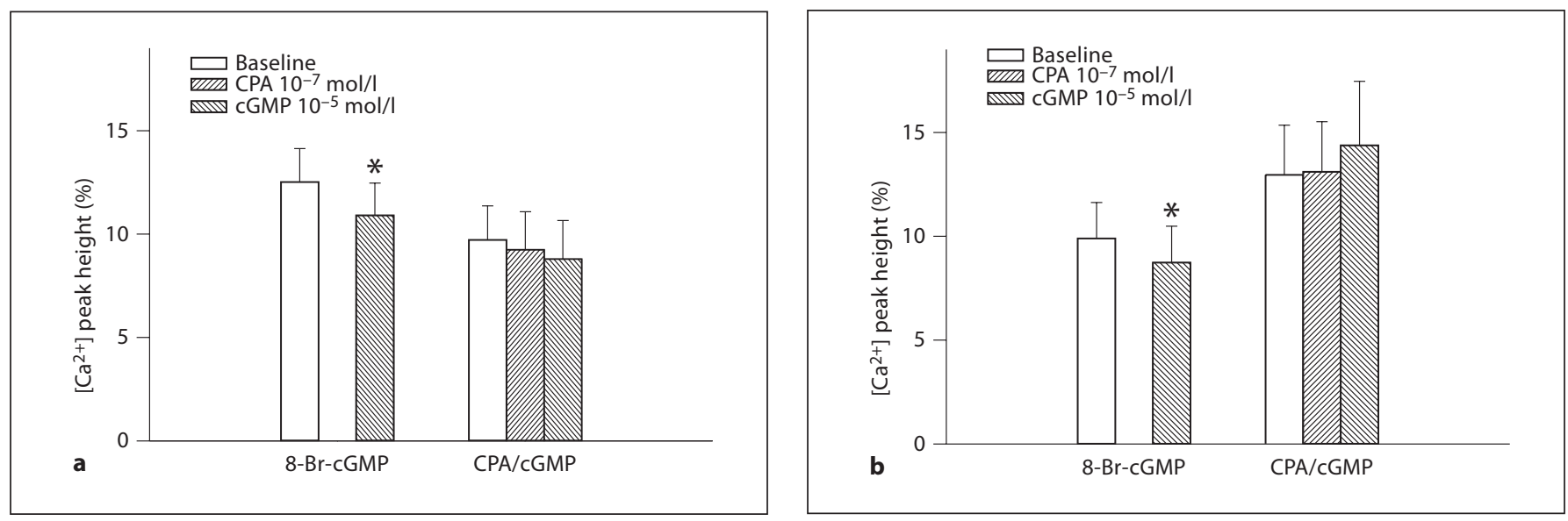

Fig. 4. The effects of 8-Br-cGMP on intracellular $\mathrm{Ca}^{2+}$ transients in control (a) and $1 \mathrm{~K} 1 \mathrm{C}$ ventricular myocytes (b). 8 -Br-cGMP $\left(10^{-5} \mathrm{~mol} / \mathrm{l}\right)$ reduced the peak calcium transients in both groups. This effect was blocked by CPA. * Significantly different from baseline.

ing and relaxation, but did not prevent the reduction in percent shortening.

In $1 \mathrm{~K} 1 \mathrm{C}$ hypertrophic ventricular myocytes, both $\mathrm{CPA}$ and thapsigargin reduced the negative effects of 8 Br-cGMP on myocyte contractility. 8-Br-cGMP no longer significantly reduced percent shortening or maximum rate of contraction following thapsigargin or CPA (fig. 1, 2). There were no significant effects of CPA or thapsigargin on maximum rate of relaxation at baseline or following the administration of 8-Br-cGMP (fig. 3).
SERCA Inhibition Prevented Reductions in Intracellular $\mathrm{Ca}^{2+}$ Transients in Response to 8-BrcGMP in Both Control and Hypertrophic Myocytes $\mathrm{Ca}^{2+}$ transients were measured in control and hypertrophied myocytes at baseline and after 8-Br-cGMP in presence or absence of CPA. As shown in figure 4, 8-BrcGMP $\left(10^{-5} \mathrm{~mol} / \mathrm{l}\right)$ significantly decreased the amplitude of the $\mathrm{Ca}^{2+}$ transients in both control and hypertrophic myocytes. In control myocytes treated with 8-Br-cGMP $10^{-5} \mathrm{~mol} / \mathrm{l}$, the $\mathrm{Ca}^{2+}$ transient peaks decreased $19 \%$ from $12.5 \pm 1.6$ to $9.9 \pm 1.6 \%$. In hypertrophic myocytes, 8 - 
Table 1. Effects of 8-Br-cGMP on intracellular $\mathrm{Ca}^{2+}$ transient timing parameters (s) in control and $1 \mathrm{~K} 1 \mathrm{C}$ ventricular myocytes in the presence and absence of CPA

\begin{tabular}{lllll}
\hline & $\begin{array}{l}\text { Control time } \\
\text { to 50\% peak }\end{array}$ & $\begin{array}{l}\text { Control time } \\
\text { to 50\% return }\end{array}$ & $\begin{array}{l}\text { 1K1C time } \\
\text { to 50\% peak }\end{array}$ & $\begin{array}{l}\text { 1K1C time } \\
\text { to 50\% return }\end{array}$ \\
\hline Baseline & $0.09 \pm 0.04$ & $0.14 \pm 0.05$ & $0.08 \pm 0.09$ & $0.13 \pm 0.05$ \\
8-Br-cGMP & $0.11 \pm 0.04$ & $0.17 \pm 0.06^{*}$ & $0.11 \pm 0.05^{*}$ & $0.18 \pm 0.05^{*}$ \\
Baseline & $0.15 \pm 0.05$ & $0.22 \pm 0.07$ & $0.09 \pm 0.05$ & $0.15 \pm 0.05$ \\
CPA & $0.15 \pm 0.05$ & $0.21 \pm 0.07$ & $0.09 \pm 0.06$ & $0.15 \pm 0.06$ \\
8-Br-cGMP & $0.16 \pm 0.06$ & $0.20 \pm 0.07$ & $0.08 \pm 0.06$ & $0.15 \pm 0.05$ \\
\hline
\end{tabular}

Values are presented as mean \pm SEM. ${ }^{*}$ Significantly different from baseline.

Br-cGMP also reduced the percentage peak height of fluorescence intensity, but to a lesser extent (11\%) from $9.9 \pm 1.7$ to $8.7 \pm 1.8 \%$. CPA blocked the 8 -Br-cGMPinduced reduction in intracellular calcium levels in both control and $1 \mathrm{~K} 1 \mathrm{C}$ myocytes. In the presence of CPA and 8 -Br-cGMP, the intracellular $\mathrm{Ca}^{2+}$ percentage peak heights were not significantly different from baseline or CPA alone (fig. 4). Other data related to the calcium transient timing parameters are shown in table 1.

\section{Discussion}

In this study, we found that the addition of cyclic GMP significantly decreased cell function and calcium transients in ventricular myocytes from control rabbit hearts. Cyclic GMP also decreased function and calcium transients in myocytes from the $1 \mathrm{~K} 1 \mathrm{C}$ hypertrophic hearts, but to a lesser extent. Sarcoplasmic reticulum $\mathrm{Ca}^{2+}$ ATPase inhibition did not affect baseline function in either group. One major finding of this study was that the effects of cyclic GMP in reducing myocyte function were partially mediated through reducing SERCA activity. The other major finding was that the interaction between SERCA and cyclic GMP did not explain the reduced effects of cyclic GMP in hypertrophic myocytes. In the presence of the SERCA inhibitors CPA or thapsigargin the effects of cyclic GMP on myocyte function were blunted in both control and hypertrophic myocytes. This suggests that SERCA plays an important role in mediating the effects of cyclic GMP in both control and hypertrophic myocytes.

Cyclic GMP, a signaling molecule common to natriuretic peptides and nitric oxide, plays an important role in the control of myocardial function. It has been shown that cyclic GMP can reduce myocardial metabolism, inotropy and function $[11,18,19]$. The negative functional effects of cyclic GMP are mainly mediated through the cyclic GMP-dependent protein kinase and this can reduce intracellular $\mathrm{Ca}^{2+}$ by activation of SERCA $[7,8]$. The effects of cyclic GMP on SERCA appear to be related to cyclic GMP-mediated phosphorylation of phospholamban [20]. Unphosphorylated phospholamban inhibits SERCA activity by decreasing the affinity of the ATPase for $\mathrm{Ca}^{2+}$. Phosphorylation of phospholamban relieves SERCA inhibition and increases SERCA activity. Cyclic GMP can also regulate calcium slow channels (L-type) in the cardiac muscle $[1,21]$. The cyclic GMP signaling pathway may also be mediated by cyclic GMP protein kinaseindependent interactions with other molecules in the cell, such as cyclic GMP-gated cation channels and certain phosphodiesterases [10, 22]. In the current study, we demonstrated that increasing the intracellular level of cyclic GMP with 8-Br-cGMP reduced ventricular myocyte function and calcium transients in control cells.

Inhibitors of SERCA such as CPA and thapsigargin have been used to block $\mathrm{Ca}^{2+}$ uptake by SERCA into the sarcoplasmic reticulum and to maintain intracellular cytosolic $\mathrm{Ca}^{2+}$ levels [23]. These agents can dramatically affect calcium transients in cardiac myocytes. In this study, we showed that negative effects of cyclic GMP on ventricular myocyte function were reduced in the presence of the SERCA inhibitors thapsigargin or CPA, indicating that cyclic GMP-induced reductions in cardiac myocyte function were at least partially mediated through the action of SERCA. This interaction between cyclic GMP and SERCA had been demonstrated in previous studies $[7,8$, 20]. We also provided direct evidence (fig. 4; table 1) that intracellular calcium transients in cardiac myocytes decreased upon addition of cyclic GMP to the cells.

Cardiac hypertrophy develops as an adaptive and compensatory mechanism in response to chronic increase in hemodynamic pressure overload. If the stress continues, there can be a transition to heart failure [24, 25]. Two major factors, mechanical stress and neural/ hormone levels, stimulate intracellular signaling pathways, alter gene expression, and result in increases in cardiac myocyte size, extracellular matrices, and heart mass. The molecular composition of the failing heart is altered in various aspects including contractile proteins, $\mathrm{Ca}^{2+} \mathrm{cy}-$ cling, signal transduction pathways, metabolism, endocrine function, and extracellular matrix [26]. Abnormal calcium fluxes have been linked to heart malfunction in patients with heart failure and in experimental animal 
models. Decreased expression and activity of SERCA are associated with hypertrophy and heart failure [27]. Renal hypertension can cause many direct and indirect changes in myocardial function and lead to cardiovascular morbidity and mortality [28]. The 1K1C animal model has been used to produce hypertension and heart hypertrophy, to study associated structural and functional changes and to investigate prevention and treatment [29, 30]. Previous studies have shown increases in the heart weight to body weight ratio, in myocyte size and an elevated systolic blood pressure in $1 \mathrm{~K} 1 \mathrm{C}$ rabbits $[16,31]$. Myocardial oxygen consumption and coronary blood flow were higher in this form of cardiac hypertrophy [32]. In this compensated form of cardiac hypertrophy, $1 \mathrm{~K} 1 \mathrm{C}$ ventricular myocyte function was similar to control cells under baseline conditions.

The intracellular cyclic GMP level in hypertrophic hearts varies dependent on the animal model used. In a canine pressure-overload hypertrophy model, cyclic GMP levels were significantly increased [15]. Other reports showed that cyclic GMP levels in rat hearts with aortic constriction were not elevated [33]. Previous work in our laboratory found that intracellular cyclic GMP level and guanylyl cyclase activity in $1 \mathrm{~K} 1 \mathrm{C}$ rabbit hearts were similar to normal hearts $[16,31]$. Nitric oxide, which stimulates cyclic GMP production, can also act as a negative regulator of cardiomyocytehypertrophy [11]. Mechanisms for the inhibitory effect of nitric oxide and cyclic GMP on cardiomyocyte hypertrophy are not well understood. Gene transfer of cyclic GMP-dependent protein kinase I enhanced the antihypertrophic effects of nitric oxide on cardiomyocyte hypertrophy [11].

In the current study, we found that adding exogenous cyclic GMP reduced ventricular myocyte function. We found 8 -Br-cGMP reduced percent shortening, maximum rate of contraction and maximum rate of relaxation. The effects of cyclic GMP were significantly blunted in the $1 \mathrm{~K} 1 \mathrm{C}$ cardiac myocytes. Previous studies had suggested reduced cyclic GMP-dependent protein kinase activity in this and other models of cardiac hypertrophy $[5,16,17]$. There may also be reductions in the activity of some of the cyclic GMP-affected cyclic AMP phosphodiesterases in myocardial hypertrophy [4]. We found that two different SERCA inhibitors, thapsigargin and CPA, prevented the negative functional effects of cyclic GMP in both control and $1 \mathrm{~K} 1 \mathrm{C}$ hypertrophic ventricular myocytes. This suggests that the interaction of SERCA and the second messenger cyclic GMP remains intact in the $1 \mathrm{~K} 1 \mathrm{C}$ myocyte.

Cyclic GMP, SERCA and Cardiac Hypertrophy
In this study, we also investigated changes in the calcium transients caused by cyclic GMP in control and hypertrophic myocytes. In both groups, 8-Br-cGMP reduced the peak height of the calcium transients, although this effect was greater in the control cells. We found that blocking SERCA activity with CPA reduced the effects of cyclic GMP on myocyte contractility and calcium transients in both control and 1K1C myocytes. This demonstrated that the negative functional effects of cyclic GMP were related to reductions in the peak of the calcium in control and hypertrophic myocytes. However, the reduced functional effects of cyclic GMP in 1K1C myocytes were still blocked by inhibition of SERCA. This suggested that other factors that cyclic GMP affects, such as changes in cyclic GMP protein kinase or cyclic GMP-affected cyclic AMP phosphodiesterases, were important $[4,5,16$, 17]. It also suggested that the effects of cyclic GMP on phospholamban [20], which have major effects on SERCA activity, were not significantly altered in the $1 \mathrm{~K} 1 \mathrm{C}$ hypertrophic ventricular myocyte. Further work is necessary to determine the cause of the decrement in the effect of cyclic GMP in cardiac hypertrophy.

In summary, we showed that cyclic GMP exerted negative effects on function and calcium transients in both control and hypertrophic myocytes. However, these effects were blunted in the $1 \mathrm{~K} 1 \mathrm{C}$ ventricular myocytes. These effects of cyclic GMP could be blunted in the presence of the SERCA blockers CPA or thapsigargin in hypertrophic as well as in control myocytes. Thus, the interaction between cyclic GMP and SERCA was preserved in hypertrophic cardiac myocytes. The decrease in response to cyclic GMP in hypertrophic myocytes was not due to malfunction of SERCA. SERCA also played an important role in cyclic GMP-mediated signaling in hypertrophic cardiac myocytes.

\section{Acknowledgment} 40320

This study was supported, in part, by USPHS Grant HL

References

Pharmacology 2009;83:223-230 
-3 Su J, Zhang Q, Moalem J, Tse J, Scholz PM, Weiss HR: Functional effects of C-type natriuretic peptide and nitric oxide are attenuated in hypertrophic myocytes from pressure-overloaded mouse hearts. Am J Physiol Heart Circ Physiol 2005;288:H1367-H1373.

4 Weiss HR, Gong GX, Straznicka M, Yan L, Tse J, Scholz PM: Cyclic GMP and cyclic AMP induced changes in control and hypertrophic cardiac myocyte function interact through cyclic GMP affected cyclic-AMP phosphodiesterases. Can J Physiol Pharmacol 1999;77:422-431.

5 Yan L, Zhang Q, Scholz PM, Weiss HR: Cyclic GMP protein kinase activity is reduced in thyroxine-induced hypertrophic cardiac myocytes. Clin Exp Pharmacol Physiol 2003; 30:943-950.

$\checkmark 6$ Zhang Q, Lazar M, Molino B, Rodriguez R, Davidov T, Su J, Tse J, Weiss HR, Scholz PM: Reduction in interaction between cGMP and cAMP in dog ventricular myocytes with hypertrophic failure. Am J Physiol Heart Circ Physiol 2005;289:H1251-H1257.

7 Lau KL, Kong SK, Ko WH, Kwan HY, Huang Y, Yao X: cGMP stimulates endoplasmic reticulum $\mathrm{Ca}^{2+}$-ATPase in vascular endothelial cells. Life Sci 2003;73:2019-2028.

$\checkmark 8$ Zhang Q, Yan L, Weiss HR, Scholz PM: Cyclic GMP-induced reduction in cardiac myocyte function is partially mediated by activation of the sarcoplasmic reticulum $\mathrm{Ca}^{2+}$ ATPase. Pharmacology 2002;64:106-112.

$\checkmark 9$ Nascimento JH, Salle L, Hoebeke J, Argibay J, Peineau N: cGMP-mediated inhibition of cardiac L-type $\mathrm{Ca}^{2+}$ current by a monoclonal antibody against the $\mathrm{M}_{2}$ ACh receptor. Am J Physiol Cell Physiol 2001;281:C1251-C1258.

-10 Vila-Petroff MG, Younes A, Egan J, Lakatta EG, Sollott SJ: Activation of distinct cAMPdependent and cGMP-dependent pathways by nitric oxide in cardiac myocytes. Circ Res 1999;84:1020-1031.
11 Wollert KC, Fiedler B, Gambaryan S, Smolenski A, Heineke J, Butt E, Trautwein C, Lohmann SM, Drexler H: Gene transfer of cGMP-dependent protein kinase I enhances the antihypertrophic effects of nitric oxide in cardiomyocytes. Hypertension 2002;39: 87-92.

12 Hardt SE, Sadoshima J: Negative regulators of cardiac hypertrophy. Cardiovasc Res 2004;63:500-509.

13 Vakili BA, Okin PM, Devereux RB: Prognostic implications of left ventricular hypertrophy. Am Heart J 2001;141:334-341.

14 Nichols JR, Gonzalez NC: Increase in myocardial cell cGMP concentration in pressure-induced myocardial hypertrophy. J Mol Cell Cardiol 1982;14:181-183.

15 Roitstein A, Kedem J, Cheinberg B, Weiss HR, Tse J, Scholz PM: The effect of intracoronary nitroprusside on cyclic GMP and regional mechanics is altered in a canine model of left ventricular hypertrophy. J Surg Res 1994;57:584-590.

-16 Straznicka M, Gong G, Yan L, Scholz PM, Weiss HR: Cyclic GMP protein kinase mediates negative metabolic and functional effects of cyclic GMP in control and hypertrophied rabbit cardiac myocytes. J Cardiovasc Pharmacol 1999;34:229-236.

-17 Engel S, Yan L, Weiss H, Scholz P: Negative functional effects of cGMP mediated by cGMP protein kinase are reduced in T4 cardiac myocytes. Eur J Pharmacol 2001;421: 23-30.

18 Kuhn M: Molecular physiology of natriuretic peptide signalling. Basic Res Cardiol 2004; 99:76-82.

19 Wollert KC, Drexler H: Regulation of cardiac remodeling by nitric oxide: focus on cardiac myocyte hypertrophy and apoptosis. Heart Fail Rev 2002;7:317-325.

20 Zhang Q, Scholz PM, He Y, Tse J, Weiss HR: Cyclic GMP signaling and regulation of SERCA activity during cardiac myocyte contraction. Cell Calcium 2005;37:259-266.

-21 Haddad GE, Sperelakis N, Bkaily G: Regulation of the calcium slow channel by cyclic GMP dependent protein kinase in chick heart cells. Mol Cell Biochem 1995;148:8994.
22 Shah AM, MacCarthy PA: Paracrine and autocrine effects of nitric oxide on myocardial function. Pharmacol Ther 2000;86:49-86.

23 Inesi G, Hua S, Xu C, Ma H, Seth M, Prasad AM, Sumbilla C: Studies of $\mathrm{Ca}^{2+}$ ATPase (SERCA) inhibition. J Bioenerg Biomembr 2005;37:365-368

-24 Hannan RD, Jenkins A, Jenkins AK, Brandenburger Y: Cardiac hypertrophy: a matter of translation. Clin Exp Pharmacol Physiol 2003;30:517-527.

25 Lorell BH, Carabello BA: Left ventricular hypertrophy: pathogenesis, detection, and prognosis. Circulation 2000;102:470-479.

26 Ritter O, Neyses L: The molecular basis of myocardial hypertrophy and heart failure. Trends Mol Med 2003;9:313-321.

27 Babu GJ, Periasamy M: Transgenic mouse models for cardiac dysfunction by a specific gene manipulation. Methods Mol Med 2002; 112:365-377.

28 Campese VM, Krol E: Neurogenic factors in renal hypertension. Curr Hypertens Rep 2002;4:256-260.

29 Chen YH, Yet SE, Perrella MA: Role of heme oxygenase-1 in the regulation of blood pressure and cardiac function. Exp Biol Med 2003;228:447-453.

30 Perrella MA, Yet SF: Role of heme oxygenase1 in cardiovascular function. Curr Pharm Des 2003;9:2479-2487.

-31 Rabindranauth P, Naim KL, Scholz PM, Tse J, Sadoff JD, Weiss HR: Negative metabolic effects of cGMP are altered in renal hypertension induced cardiac hypertrophy. Basic Res Cardiol 1997;92:8-16.

32 Cimini CM, Upsher ME, Weiss HR: Myocardial $\mathrm{O}_{2}$ supply and consumption in early cardiac hypertrophy of renal hypertensive rabbits. Basic Res Cardiol 1989;84:13-21.

33 Dowell RT, Haithcoat JL, Thirkill HM, Palmer WK: Heart cyclic nucleotide responses to sustained aortic constriction in neonatal and adult rat. Am J Physiol 1984; 246:197-206. 